\title{
Transferable utility games with uncertainty
}

Citation for published version (APA):

Habis, H., \& Herings, P. J. J. (2011). Transferable utility games with uncertainty. Journal of Economic Theory, 146(5), 2126-2139. https://doi.org/10.1016/j.jet.2011.06.005

Document status and date:

Published: 01/09/2011

DOI:

10.1016/j.jet.2011.06.005

Document Version:

Publisher's PDF, also known as Version of record

\section{Document license:}

Taverne

\section{Please check the document version of this publication:}

- A submitted manuscript is the version of the article upon submission and before peer-review. There can be important differences between the submitted version and the official published version of record.

People interested in the research are advised to contact the author for the final version of the publication, or visit the DOI to the publisher's website.

- The final author version and the galley proof are versions of the publication after peer review.

- The final published version features the final layout of the paper including the volume, issue and page numbers.

Link to publication

\footnotetext{
General rights rights.

- You may freely distribute the URL identifying the publication in the public portal. please follow below link for the End User Agreement:

www.umlib.nl/taverne-license

Take down policy

If you believe that this document breaches copyright please contact us at:

repository@maastrichtuniversity.nl

providing details and we will investigate your claim.
}

Copyright and moral rights for the publications made accessible in the public portal are retained by the authors and/or other copyright owners and it is a condition of accessing publications that users recognise and abide by the legal requirements associated with these

- Users may download and print one copy of any publication from the public portal for the purpose of private study or research.

- You may not further distribute the material or use it for any profit-making activity or commercial gain

If the publication is distributed under the terms of Article $25 \mathrm{fa}$ of the Dutch Copyright Act, indicated by the "Taverne" license above, 


\title{
Transferable utility games with uncertainty
}

\author{
Helga Habis ${ }^{\mathrm{a}, 1}$, P. Jean-Jacques Herings ${ }^{\mathrm{b}, *, 2}$ \\ a Institute of Economics, Hungarian Academy of Sciences, and Department of Microeconomics, \\ Corvinus University of Budapest, Fövám tér 8, 1093, Budapest, Hungary \\ ${ }^{\mathrm{b}}$ Department of Economics, Maastricht University, P.O. Box 616, 6200 MD, Maastricht, The Netherlands
}

Received 1 November 2010; final version received 12 November 2010; accepted 13 May 2011

Available online 30 June 2011

\begin{abstract}
We introduce the concept of a TUU-game, a transferable utility game with uncertainty. In a TUU-game there is uncertainty regarding the payoffs of coalitions. One out of a finite number of states of nature materializes and conditional on the state, the players are involved in a particular transferable utility game. We consider the case without ex ante commitment possibilities and propose the Weak Sequential Core as a solution concept. We characterize the Weak Sequential Core and show that it is non-empty if all ex post TU-games are convex.

(C) 2011 Elsevier Inc. All rights reserved.
\end{abstract}

JEL classification: $\mathrm{C} 71 ; \mathrm{C} 73$

Keywords: Transferable utility games; Uncertainty; Weak Sequential Core

\section{Introduction}

The vast majority of cooperative game theory has focused on games with deterministic payoffs. Nevertheless, uncertainty plays an inevitable role in most surplus sharing problems. In this paper we introduce transferable utility games with uncertainty, called TUU-games. A TUUgame consists of two time periods, 0 and 1 . Period 0 is a time period before the resolution of

\footnotetext{
* Corresponding author.

E-mail addresses: helga.habis@uni-corvinus.hu (H. Habis), P.Herings@ maastrichtuniversity.nl (P.J.J. Herings).

1 The author would like to thank the Hungarian Academy of Sciences for the financial support under the Momentum Programme (LD-004/2010).

2 The author would like to thank the Netherlands Organization for Scientific Research (NWO) for financial support.
} 
uncertainty. In period 1 one out of a finite number of states of nature materializes and conditional on the state, the players are involved in a particular transferable utility game. An allocation therefore specifies a payoff to each player conditional on each possible state of nature. A utility function is then used to assign a utility level to each profile of state-contingent payoffs.

This new set-up provides a more general treatment of uncertainty than the approach that has appeared in the literature so far. Granot [5] introduced a cooperative game where the values of the coalitions are random variables with given distribution functions, and players are risk-neutral. This treatment is less complete since it specifies only the marginal distribution of the worths of coalitions, whereas our approach allows for the complete specification of the distribution, implying that for instance correlation between the worths of several coalitions can be incorporated. Suijs and Borm $[13,14]$ no longer assume risk neutrality, but keep the specification where only marginal distributions of worths are given. Bossert et al. [2] consider a pair of TU-games, one of which will be the true game. They do not use utility functions but perform a worst-case analysis. Closest to our set-up is Predtetchinski [8], where the non-transferable utility case is studied in an infinite horizon setting. His approach is similar to ours in the sense that the game to be played is determined by the particular realization of the state of nature.

The introduction of uncertainty into cooperative games raises many new and interesting issues. When players can make state-contingent agreements before the resolution of uncertainty, i.e. at the ex ante stage, period 0 , the situation boils down to a non-transferable utility game, and we can apply for instance the classical concept of the Core to determine allocations of payoffs that are stable.

We, on the contrary, are interested in the case where no binding agreements are possible before the state of nature is known, but where players have the option to discuss agreements in period 0 . Lacking the possibility to make binding agreements concerning the ex post stage, period 1, such agreements have to be self-enforcing. Our motivation for period 0 is thereby similar to the one underlying the notion of Coalition-Proof Nash equilibrium, where players discuss the strategies they are going to use, but cannot make binding commitments, and their choices have to be selfenforcing. After the resolution of uncertainty in period 1, players know the TU-game that they play and can make binding commitments as is usual in cooperative game theory.

A consequence of the absence of binding agreements in period 0 is that many ex ante desirable transfers of payoffs across states are not feasible. Indeed, in the absence of binding agreements in period 0 , only allocations in the Core of the transferable utility game that results after the state of nature is known, are self-enforcing.

Throughout the paper we assume symmetric information. There is also a rather extensive literature on cooperation in economies with private information, where usually a third stage between the ex ante and ex post stages is distinguished, namely an interim stage in which the agents learn their private information. See for instance [15] for a study of the core in this framework. In these models, however, subject to incentive compatibility constraints, a complete contracting environment results; for the special case of symmetric information one is lead to the classical core. The study of asymmetric information issues in an incomplete contracting framework remains an interesting subject for further research.

We are interested in the appropriate definition of the Core in a TUU-game. In this setting coalitions are allowed to form in both periods. Stability requires that a suggested allocation cannot be blocked by any coalition at any period, i.e. both before and after the resolution of uncertainty. We concentrate on agreements which are self-enforcing in the sense that a coalition can only deviate from a given allocation if no sub-coalition ever has a credible counter-deviation. Ray [10] shows that in a static environment the set of deviations coincides with the set of credible deviations. 
This is no longer true in our setting, and leads to the solution concept of the Weak Sequential Core.

The Weak Sequential Core was introduced in [7] for finite deterministic sequences of TUgames, and it was defined for two-period exchange economies with incomplete markets in [9]. In [7] the Weak Sequential Core was defined as the set of feasible payoff allocations for the grand coalition, from which no coalition ever has a credible deviation. In [6] it is demonstrated that the definition of credibility in [7] has to be adapted in order to show that the Weak Sequential Core has a nice characterization in terms of the cores of appropriately defined subgames. In [9] this characterization was used as the definition of the Weak Sequential Core in a two-period exchange economy; i.e. the issue of the credibility of deviations is neglected there.

We extend the notion of credible deviation of [6] to TUU-games and show that an allocation belongs to the Weak Sequential Core only if conditional on the state of nature it belongs to the Core of the related ex post TU-game. This result follows from the absence of credible deviations in period 1 . The absence of credible deviations in period 0 is then used to show that an allocation belongs to the Weak Sequential Core if moreover there is no coalition in period 0 that can propose state-contingent Core elements of the ex post games restricted to that coalition, which gives each of its members higher expected utility. In this way we obtain a characterization of the Weak Sequential Core. This characterization is in the spirit of the one proposed by [7] and later proved properly by [6], extended to the case with uncertainty.

A problem of the Weak Sequential Core concept is that the existing literature has failed to provide a general non-emptiness result, whereas moreover both Kranich et al. [7] and Predtetchinski et al. [9] give examples where the Weak Sequential Core is empty. We provide a general result on the non-emptiness of the Weak Sequential Core of TUU-games. We show that if all the ex post TU-games are convex, then the Weak Sequential Core is non-empty. This result does not impose any assumptions on the utility functions of the players beyond continuity and state-separability. We also provide examples to show that the convexity condition cannot be weakened to permutational convexity, not even when the permutation is the same for all ex post TU-games, nor can it be weakened to exactness.

The outline of the paper is as follows. We specify the model in Section 2 and give the formal definition of the Weak Sequential Core in Section 3, followed by its characterization in Section 4. We show the non-emptiness result in Section 5 and present the examples showing that permutational convexity or exactness are not sufficient for non-emptiness. Section 6 concludes.

\section{Preliminaries}

Consider a game with two time periods, $t \in T=\{0,1\}$. Period 0 corresponds to an ex ante stage before the resolution of uncertainty. In period 1 one state $s$ out of a finite set of states of nature $\{1, \ldots, S\}$ occurs. Since no confusion can arise, we also denote this set by $S$. We define the state of nature for period 0 as state 0 , so the set of all states is $S^{\prime}=\{0\} \cup S$. In period 1 the players are involved in a cooperative game with transferable utility, or briefly TU-game, where the game itself is allowed to be state-dependent.

The TU-game $\Gamma_{s}$ played in state $s \in S$ is a pair $\left(N, v_{s}\right)$, where $N=\{1,2, \ldots, n\}$ is the set of players and $v_{s}: 2^{N} \rightarrow \mathbb{R}$ is a characteristic function which assigns to each coalition $C \subset N$ its worth $v_{S}(C)$, with the convention that $v_{S}(\emptyset)=0$. The collection of non-empty subsets of $N$ is denoted by $\mathcal{N}$, so $\mathcal{N}=2^{N} \backslash\{\emptyset\}$. Player $i \in N$ evaluates his payoffs by a utility function $u^{i}$ : $\mathbb{R}^{S} \rightarrow \mathbb{R}$, which assigns to every profile of payoffs $x^{i}=\left(x_{1}^{i}, \ldots, x_{S}^{i}\right) \in \mathbb{R}^{S}$ a utility level $u^{i}\left(x^{i}\right)$. The utility function is assumed to be continuous and state-separable, i.e. $u^{i}\left(x^{i}\right)=\sum_{s \in S} u_{s}^{i}\left(x_{s}^{i}\right)$, 
where $u_{s}^{i}\left(x_{s}^{i}\right)$ is monotonically increasing. Von Neumann-Morgenstern utility functions are a prominent example of utility functions satisfying these assumptions.

A TU-game with uncertainty is defined as follows.

Definition 2.1. A $T U$-game with uncertainty (TUU-game) $\Gamma$ is a tuple $(N, S, v, u)$ where $v=\left(v_{1}, \ldots, v_{S}\right)$ are state-dependent characteristic functions and $u=\left(u^{1}, \ldots, u^{n}\right)$ are utility functions.

Note that there are no payoffs in state 0 . State 0 is merely introduced as a point in time when the players face the uncertainty in the future and may decide to agree upon future state-contingent payoff allocations. Payoffs in state 0 could be incorporated into our model but our main interest is to get insight into the effect of future uncertainty on the stability of payoff allocations.

Another observation is that when the cardinality of $S$ is one, the concept of a TUU-game collapses with the one of a TU-game. In the absence of uncertainty, all monotonic transformations of utility functions are equivalent, and it is without loss of generality to take $u^{i}\left(x^{i}\right)=x^{i}$. Our interest is obviously in the cases with non-degenerate uncertainty.

The central question in a TUU-game is how the worth $v_{S}(N)$ of the grand coalition is distributed among its members in every state $s \in S$. A distribution of worth, represented by a matrix $x=\left(x^{1}, \ldots, x^{n}\right) \in \mathbb{R}^{S \times N}$, is called an allocation. The state-s component $x_{s}=$ $\left(x_{s}^{1}, \ldots, x_{s}^{n}\right) \in \mathbb{R}^{N}$ of an allocation is referred to as the allocation in state $s \in S$. The total worth obtained by coalition $C$ in state $s$ is $x_{s}(C)=\sum_{i \in C} x_{s}^{i}$. An allocation for a coalition $C$ is a matrix $x^{C}=\left(x^{i}\right)_{i \in C} \in \mathbb{R}^{S \times C}$, with a state-s component $x_{s}^{C} \in \mathbb{R}^{C}$. The restriction of a TUU-game $\Gamma$ to coalition $C$ is a TUU-game itself and is denoted by $(\Gamma, C)$.

\section{The Weak Sequential Core}

We study which allocations in the game $\Gamma$ are stable. In general, $\bar{x}$ is stable if there is no state $s^{\prime} \in S^{\prime}$ and no coalition $C \subset N$ which has a profitable deviation from $\bar{x}$ at state $s^{\prime}$. There are various ways in which the notion of profitable deviation might be formulated. Here we concentrate on the Weak Sequential Core, introduced in [7] for finite deterministic sequences of TU-games and in [9] for two-period exchange economies with incomplete markets. Here we define the Weak Sequential Core for TUU-games.

When the classical definition of the Core [4] is adapted to situations with time and uncertainty, it is typically assumed that agents can fully commit to any state-contingent allocation. In this case one would define the set of feasible allocations for a coalition $C \subset N$ as

$$
X^{C}=\left\{x^{C} \in \mathbb{R}^{S \times C} \mid x^{C}(C) \leqslant v(C)\right\},
$$

resulting in the set of utilities for coalition $C$ given by

$$
V(C)=\left\{\bar{u}^{C} \in \mathbb{R}^{C} \mid \exists x^{C} \in X^{C}, \forall i \in C, \bar{u}^{i}=u^{i}\left(x^{i}\right)\right\},
$$

thereby obtaining an NTU-game. Full commitment may be a strong and unrealistic assumption in the presence of time and uncertainty. Once the state of nature is known, there are typically players which have no incentives to stick to the previously arranged allocation of payoffs. Here we analyze the case with absence of commitments and look for agreements which are self-enforcing.

First we define what allocations, and thereby deviations, are feasible for coalitions at different states, then we formalize the notion of credible deviations and finally we define the Weak Sequential Core of a TUU-game. We start with feasibility at future states. 
Definition 3.1. Fix some allocation $\bar{x}$. The allocation $x^{C}$ is feasible for coalition $C$ at state $s \in S$ given $\bar{x}$ if

$$
\begin{aligned}
& x_{-s}^{C}=\bar{x}_{-s}^{C}, \\
& x_{s}^{C}(C) \leqslant v_{s}(C) .
\end{aligned}
$$

The first condition requires that the members of a coalition take allocations outside state $s$ as given. Since utility functions are assumed to be state-separable, this assumption is harmless. According to the second condition, in state $s$ the members of a coalition can redistribute at most their worth.

We turn next to feasibility as state 0 .

Definition 3.2. The allocation $x^{C}$ is feasible for a coalition $C$ at state 0 if

$$
x^{C}(C) \leqslant v(C) \text {. }
$$

Note that feasibility at state 0 requires that the allocation must be feasible for coalition $C$ in every state; it requires $\sum_{i \in C} x_{s}^{i} \leqslant v_{s}(C)$ to hold for all states $s$ in period 1.

We continue by defining deviations as feasible allocations that improve the utility of every coalition member.

Definition 3.3. Fix some allocation $\bar{x}$. A coalition $C$ can deviate from $\bar{x}$ at state $s^{\prime} \in S^{\prime}$ if there exists a feasible allocation $x^{C}$ for $C$ at $s^{\prime}$ given $\bar{x}$ such that

$$
u^{i}\left(x^{i}\right)>u^{i}\left(\bar{x}^{i}\right), \quad \text { for all } i \in C .
$$

The allocation $x^{C}$ in Definition 3.3 is referred to as a deviation. Definition 3.3 can be extended in an obvious way to define deviations from an allocation $x^{C}$ by a sub-coalition $D$ of $C$.

We show in the following example that deviations are not necessarily self-enforcing.

Example 3.4. Consider a TUU-game with two players and with two states in period 1 with equal probability of occurrence. The players are assumed to be strictly risk-averse expected utility maximizers. Let the state-dependent characteristic function be the following: $v_{1}(\{1,2\})=$ $v_{2}(\{1,2\})=1, v_{1}(\{1\})=v_{2}(\{2\})=1, v_{1}(\{2\})=v_{2}(\{1\})=0$. The characteristic function has the feature that player $i$ does not contribute to the surplus at state $s=-i$.

Let the allocation

$$
\bar{x}=\left(\bar{x}^{1}, \bar{x}^{2}\right)=\left(\begin{array}{ll}
1 & 0 \\
0 & 1
\end{array}\right)
$$

be given. Now consider the allocation

$$
x=\left(x^{1}, x^{2}\right)=\left(\begin{array}{cc}
\frac{1}{2} & \frac{1}{2} \\
\frac{1}{2} & \frac{1}{2}
\end{array}\right),
$$

which is feasible for the grand coalition in state 0 . Since both players are risk-averse, $x$ is a deviation from $\bar{x}$ at state 0 by coalition $\{1,2\}$.

The allocation $x$ is not self-enforcing though, since after the resolution of uncertainty it will always be blocked by a singleton coalition; at state 1 player 1 can block $x_{1}^{1}=\frac{1}{2}$ by $\hat{x}_{1}^{1}=v_{1}(\{1\})=$ 1 and at state 2 player 2 can block $x_{2}^{2}=\frac{1}{2}$ by $\hat{x}_{2}^{2}=v_{2}(\{2\})=1$. 
Since deviations should be self-enforcing, we introduce the notion of credible deviations. In defining credibility, we follow the approach developed in [10] for the static case. Ray [10] shows that in a static environment the set of deviations coincides with the set of credible deviations. This is no longer true in our setting.

Credible deviations are defined recursively and by backwards induction. At any future state, any deviation by a singleton coalition is credible. A two-player coalition has a credible deviation at a future state if there is no singleton sub-coalition with a credible counter-deviation at that state. A credible deviation at a future state for an arbitrary coalition is then defined by recursion. More formally, a recursive definition of a credible deviation at state $s \in S$ by a coalition $C$ is as follows.

Definition 3.5. Fix some allocation $\bar{x}$. Any deviation $x^{C}$ from $\bar{x}$ at state $s \in S$ by a singleton coalition is credible. Suppose credible deviations have been defined for each coalition of size $k$. Let $C$ be a coalition of size $k+1$. A deviation $x^{C}$ from $\bar{x}$ at state $s$ by coalition $C$ is credible if there is no sub-coalition $D \subsetneq C$ such that $D$ has a credible deviation from $x^{C}$ at state $s$.

At state 0 , again, any deviation by a singleton coalition is credible. A two-player coalition has a credible deviation at state 0 if there is no singleton sub-coalition with a credible counterdeviation at any state, current or future. A credible deviation at state 0 by an arbitrary coalition is then defined by recursion. More formally, we have the following definition.

Definition 3.6. Fix some allocation $\bar{x}$. Any deviation $x^{C}$ from $\bar{x}$ at state 0 by a singleton coalition is credible. Suppose credible deviations have been defined for each coalition of size $k$. Let $C$ be a coalition of size $k+1$. A deviation $x^{C}$ from $\bar{x}$ at state 0 by coalition $C$ is credible if there is no sub-coalition $D \subsetneq C$ and state $s^{\prime} \in S^{\prime}$ such that $D$ has a credible deviation from $x^{C}$ at $s^{\prime}$.

Definition 3.7. The Weak Sequential Core $\operatorname{WSC}(\Gamma)$ of the game $\Gamma$ is the set of feasible allocations $\bar{x}$ for the grand coalition from which no coalition ever has a credible deviation.

Our definition of the Weak Sequential Core is different from the one in [7] and the one in [9]. Kranich et al. [7] do not require the counter-deviation by a sub-coalition to be credible, which leads to problems as demonstrated in [6]. We adapt the definition in [6] to TUU-games. The definition of the Weak Sequential Core in [9] for an incomplete markets exchange economy is based directly on the characterization we present in Theorem 4.4. We would also like to point out the similarity of our recursive definition to the one used in the concept of Coalition-Proof Nash equilibrium [1]. In both cases the notion of self-enforcement is interpreted as the absence of deviating sub-coalitions, where the recursive approach guarantees consistency of this notion.

Example 3.4 (continued). We show that $\bar{x}$ is the only allocation which belongs to the Weak Sequential Core of the game. For an allocation $x$ to belong to the Weak Sequential Core, it must hold that $x_{1}^{1} \geqslant 1$, since otherwise player 1 could credibly block $x$ in state 1 by $\hat{x}_{1}^{1}=v_{1}(\{1\})=1$. An analogous reasoning implies that $x_{1}^{2} \geqslant 0$. Similarly, $x_{2}^{2} \geqslant 1$ must hold, since otherwise player 2 could credibly block $x$ in state 2 by $\hat{x}_{2}^{2}=v_{2}(\{2\})=1$, and by analogous reasons we have $x_{2}^{1} \geqslant 0$. Now it follows from feasibility for the grand coalition that $\bar{x}$ is the only candidate element of WSC $(\Gamma)$.

Clearly, singleton coalitions cannot deviate from $\bar{x}$ at any state. The same is obviously true for the grand coalition at any future state. The arguments already used to derive that $\bar{x}$ is the only 
candidate as a Weak Sequential Core element, imply that the grand coalition does not have a credible deviation from $\bar{x}$ at state 0 .

\section{Characterization}

In this section we provide a useful characterization for the Weak Sequential Core. Consider a particular credible deviation at state 0 by some coalition. We show that the set consisting of all credible deviations which improve the utility of all coalition members by the same amount or more is a compact set.

Lemma 4.1. Let $\bar{x}$ be a feasible allocation and let $\hat{x}^{C}$ be a credible deviation from $\bar{x}$ at state 0 by a coalition $C$ of size greater than or equal to two. Let $X$ be the set of credible deviations $x^{C}$ from $\bar{x}$ at state 0 by coalition $C$ such that $u^{i}\left(x^{i}\right) \geqslant u^{i}\left(\hat{x}^{i}\right)$ for all $i \in C$. Then the set $X$ is compact.

Proof. First we show that $X$ is closed. Consider a sequence $\left(x_{m}^{C}\right)_{m \in \mathbb{N}}$ with $x_{m}^{C} \in X$ converging to $\tilde{x}^{C}$. We need to show that $\tilde{x}^{C} \in X$, so

(i) $\tilde{x}^{C}$ is a credible deviation from $\bar{x}$ at state 0 by $C$,

(ii) $u^{i}\left(\tilde{x}^{i}\right) \geqslant u^{i}\left(\hat{x}^{i}\right)$ for all $i \in C$.

The continuity of $u^{i}$ implies $u^{i}\left(\tilde{x}^{i}\right) \geqslant u^{i}\left(\hat{x}^{i}\right)$ for all $i \in C$, thus (ii) holds.

Clearly, $\tilde{x}^{C}$ is a deviation from $\bar{x}$ at state 0 by $C$, so if $\tilde{x}^{C}$ is not a credible deviation then there is a credible deviation $y^{D}$ from $\tilde{x}^{C}$ at $s^{\prime} \in S^{\prime}$ by a sub-coalition $D \subsetneq C$. Since $u^{i}\left(\tilde{x}^{i}\right)<u^{i}\left(y^{i}\right)$ for all $i \in D$ there must be an $\hat{m}$ such that if $m>\hat{m}$ then for all $i \in D, u^{i}\left(x_{m}^{i}\right)<u^{i}\left(y^{i}\right)$. This makes $y^{D}$ a credible deviation from $x_{m}^{C}$ at state $s^{\prime}$ by coalition $D$, a contradiction, so (i) holds. Hence, $X$ is closed.

Now we show that $X$ is bounded. For all $x^{C} \in X$ it holds that

$$
x^{i} \geqslant v(\{i\}), \quad i \in C,
$$

since no player in $C$ should have a credible deviation from $x^{C}$ at any $s \in S$. Therefore $X$ is bounded from below. Since $x^{C}(C) \leqslant v(C)$, it follows that $X$ is also bounded from above.

Note that Lemma 4.1 is not true for the set of deviations rather then the set of credible deviations, since in the case of deviations it might be possible to compensate arbitrarily negative payoffs in one state by sufficiently high positive payoffs in other states. For the same reason, Lemma 4.1 is not true for singleton coalitions, since for these coalitions deviations and credible deviations coincide.

Our characterization of the Weak Sequential Core makes use of the classical notion of the Core of a TU-game.

Definition 4.2. A coalition $C$ can improve upon an allocation $\bar{x}$ in a TU-game $(N, v)$ if $\bar{x}(C)<v(C)$.

Definition 4.3. The Core $C(N, v)$ of a TU-game $(N, v)$ is the collection of allocations $\bar{x}$ such that $\bar{x}(N)=v(N)$ and there is no coalition $C$ that can improve upon $\bar{x}$. 
The Weak Sequential Core can be characterized by means of the Core of suitably chosen subgames.

Theorem 4.4. The following two statements are equivalent:

(a) $\bar{x} \in \operatorname{WSC}(\Gamma)$,

(b) $\bar{x}$ is such that $\bar{x}_{s} \in \mathrm{C}\left(\Gamma_{s}\right)$ for all $s \in S$, and there is no $C \subset N$ and allocation $x^{C}$ such that $x_{s}^{C} \in \mathrm{C}\left(\Gamma_{s}, C\right)$ for all $s \in S$, and $u^{i}\left(x^{i}\right)>u^{i}\left(\bar{x}^{i}\right)$ for all $i \in C$.

Proof. (a) $\Rightarrow$ (b). Consider some state $s \in S$ and suppose there is a coalition $C \subset N$ that can improve upon $\bar{x}_{s}$ by $x_{s}^{C}$. We define $x_{-s}^{C}=\bar{x}_{-s}^{C}$. Either $x^{C}$ is a credible deviation from $\bar{x}$ at state $s$ by coalition $C$ or there is a sub-coalition $D \subsetneq C$ such that $D$ has a credible deviation $y^{D}$ from $x^{C}$ at $s$. In the latter case $y^{D}$ is also a credible deviation from $\bar{x}$ at state $s$ by coalition $D$. In both cases we have a contradiction with $\bar{x} \in \mathrm{WSC}(\Gamma)$. It follows that $\bar{x}_{s} \in \mathrm{C}\left(\Gamma_{s}\right)$.

Suppose there is $C \subset N$ and $x^{C}$ such that $x_{s}^{C} \in \mathrm{C}\left(\Gamma_{s}, C\right)$ for all $s \in S$, and $u^{i}\left(x^{i}\right)>u^{i}\left(\bar{x}^{i}\right)$ for all $i \in C$. We show that if such a deviation exists then there also exists a credible deviation, thereby contradicting (a). If $x^{C}$ is a credible deviation from $\bar{x}$ at 0 by $C$, then we are done, so suppose this is not the case. Since $x_{s}^{C} \in \mathrm{C}\left(\Gamma_{s}, C\right)$ holds for all $s \in S$, there cannot be a credible deviation from $x^{C}$ at $s \in S$ by some coalition $D \subsetneq C$, so there must be a credible deviation $y^{D}$ from $x^{C}$ at state 0 by some coalition $D \subsetneq C$. But then $y^{D}$ is also a credible deviation from $\bar{x}$ at state 0 by $D$ since $u^{i}\left(y^{i}\right)>u^{i}\left(x^{i}\right)>u^{i}\left(\bar{x}^{i}\right)$ for all $i \in D$.

(b) $\Rightarrow$ (a). Suppose (a) does not hold. Since $\bar{x}_{s} \in \mathrm{C}\left(\Gamma_{s}\right)$ for all $s \in S$, no coalition has a credible deviation from $\bar{x}$ at $s \in S$ and so there must be a credible deviation $\hat{x}^{C}$ from $\bar{x}$ at state 0 by a coalition $C$. We will show that then there also exists a credible deviation $\tilde{x}^{C}$ from $\bar{x}$ at state 0 by coalition $C$ such that $\tilde{x}_{s}^{C} \in \mathrm{C}\left(\Gamma_{s}, C\right)$ for all $s \in S$, thereby violating (b). When $C$ contains a single player, say $i$, we define $\tilde{x}_{s}^{C}=v_{s}(\{i\})$ for all $s \in S$ and we are done, so consider the case where $C$ is of size greater than or equal to two.

Let $X$ be the set of credible deviations $x^{C}$ from $\bar{x}$ at state 0 by $C$ with the property that $u^{i}\left(x^{i}\right) \geqslant u^{i}\left(\hat{x}^{i}\right)$ for all $i \in C$. Let $\tilde{x}^{C}$ be a solution of the problem

$$
\max _{x^{C} \in X} \sum_{i \in C} u^{i}\left(x^{i}\right) .
$$

Since the allocation $\hat{x}^{C}$ belongs to $X, X$ is non-empty. We know from Lemma 4.1 that $X$ is compact. Therefore the set of maximizers in (1) is non-empty.

We show that $\tilde{x}_{s}^{C}$ belongs to $\mathrm{C}\left(\Gamma_{s}, C\right)$ for all $s \in S$. Suppose there exists a state $s \in S$ for which $\tilde{x}_{s}^{C} \notin \mathrm{C}\left(\Gamma_{s}, C\right)$. Then there is a coalition $D \subset C$ that can improve upon $\tilde{x}_{s}^{C}$ by means of $y_{s}^{D} \gg \tilde{x}_{s}^{C}$. We define the allocation $y^{D}$ by setting $y_{-s}^{D}=\tilde{x}_{-s}^{C}$. Since $\tilde{x}^{C}$ is a credible deviation from $\bar{x}$, either $D=C$ or $D \subsetneq C$ and there is a proper sub-coalition of $D$ with a credible counterdeviation from $y^{D}$ at state $s$. The latter credible counter-deviation is a credible deviation from $\tilde{x}^{C}$ at state $s$, a contradiction. It follows that $D=C$.

We show that $y^{C}$ belongs to $X$. By the separability of the utility function it holds that $u^{i}\left(y^{i}\right)>$ $u^{i}\left(\tilde{x}^{i}\right) \geqslant u^{i}\left(\hat{x}^{i}\right)$ for all $i \in C$. Moreover, since $\tilde{x}^{C}$ is a credible deviation from $\bar{x}$ at state 0 by $C$ and $y_{s}^{C} \geqslant \tilde{x}_{s}^{C}$ for all $s \in S$, we have that $y^{C}$ is a credible deviation from $\bar{x}$ at state 0 by $C$, and it follows that $y^{C} \in X$.

We have that $\sum_{i \in C} u^{i}\left(y^{i}\right)>\sum_{i \in C} u^{i}\left(\tilde{x}^{i}\right)$, which contradicts that $\tilde{x}^{C}$ is a maximizer. We have shown that $\tilde{x}_{s}^{C} \in \mathrm{C}\left(\Gamma_{s}, C\right)$ for all $s \in S$. 
Table 1

Characteristic function.

\begin{tabular}{llrrllccc}
\hline$v$ & $\emptyset$ & $\{1\}$ & $\{2\}$ & $\{3\}$ & $\{1,2\}$ & $\{1,3\}$ & $\{2,3\}$ & $\{1,2,3\}$ \\
\hline$v_{1}$ & 0 & 5 & 50 & 10 & 140 & 20 & 140 & 150 \\
$v_{2}$ & 0 & 50 & 5 & 10 & 140 & 140 & 20 & 150 \\
\hline
\end{tabular}

For an allocation to belong to the Weak Sequential Core of the TUU-game $\Gamma$, the allocation should belong to the Core of the TU-game $\Gamma_{s}$ in every state $s \in S$. Moreover, no coalition should be able to pick an element of the Core of the game restricted to $C$ in every state, and in doing so improve utility in an ex ante sense.

It follows immediately from Theorem 4.4 that the Weak Sequential Core of a TUU-game with one state coincides with the Core of that game.

In a TUU-game one can distinguish ex ante and ex post efficiency.

Definition 4.5. An allocation $\bar{x}$ is ex ante efficient in the game $\Gamma$ if:

(i) $\bar{x}(N)=v(N)$.

(ii) There does not exist an allocation $x$ with $x(N) \leqslant v(N)$ such that $u^{i}\left(x^{i}\right)>u^{i}\left(\bar{x}^{i}\right)$ for all $i \in N$.

Definition 4.6. An allocation $\bar{x}$ is ex post efficient in the game $\Gamma$ if $\bar{x}(N)=v(N)$.

Note, that the concept of ex post efficiency says more than the usual feasibility conditions in TU-games, since it requires $\sum_{i \in N} \bar{x}_{s}^{i}=v_{s}(N)$ to hold at all states $s \in S$, but contrary to ex ante efficiency it does not imply Pareto-efficiency, since it does not consider reallocation possibilities across states.

Corollary 4.7. If $\bar{x} \in \mathrm{WSC}(\Gamma)$, then $\bar{x}$ is ex post efficient.

Observe that Example 3.4 demonstrates that an allocation in the Weak Sequential Core might not be ex ante efficient.

\section{Non-emptiness}

Kranich et al. [7] show that the Weak Sequential Core of a finite deterministic sequence of TU-games is non-empty if all utility functions are linear. Predtetchinski et al. [9] give sufficient conditions for non-emptiness for the case of an exchange economy with two agents. These are the only results in the literature so far regarding non-emptiness of the Weak Sequential Core. Both papers present examples where the Weak Sequential Core is empty.

The Weak Sequential Core can also be empty in a TUU-game, as shown in the following example.

Example 5.1. Consider a TUU-game $\Gamma$ with three players and two future states, both occurring with equal probability. The characteristic function $v$ is presented in Table 1 .

Players are strictly risk averse expected utility maximizers.

By Theorem 4.4 only allocations in the Core of $\Gamma_{1}$ and $\Gamma_{2}$ can be stable. The Core of each of these TU-games consists of exactly one vector: 


$$
\begin{aligned}
& \mathrm{C}\left(\Gamma_{1}\right)=\{(10,130,10)\}, \\
& \mathrm{C}\left(\Gamma_{2}\right)=\{(130,10,10)\} .
\end{aligned}
$$

The resulting allocation

$$
\bar{x}=\left(\bar{x}^{1}, \bar{x}^{2}, \bar{x}^{3}\right)=\left(\begin{array}{ccc}
10 & 130 & 10 \\
130 & 10 & 10
\end{array}\right)
$$

leads to high uncertainty for players 1 and 2, which could be completely eliminated if they cooperated. Coalition $\{1,2\}$ can credibly deviate from $\bar{x}$ by perfectly pooling their risks at state 0 , using

$$
x^{\{1,2\}}=\left(x^{1}, x^{2}\right)=\left(\begin{array}{ll}
70 & 70 \\
70 & 70
\end{array}\right),
$$

and so achieving a higher utility, since both players are strictly risk-averse expected utility maximizers. We have shown that $\operatorname{WSC}(\Gamma)=\emptyset$.

We show next that if $\Gamma_{s}$ is convex for all $s \in S$, then the Weak Sequential Core is non-empty. Notice that in Example 5.1 convexity is violated for both $\Gamma_{1}$ and $\Gamma_{2}$.

Definition 5.2. A TU-game $(N, v)$ is convex if for all $C \subset N$ and for all $S \subsetneq T \subset N \backslash C$ it holds that $v(S \cup C)-v(S) \leqslant v(T \cup C)-v(T)$.

Theorem 5.3. Let the TUU-game $\Gamma$ be such that $\Gamma_{s}$ is convex for all $s \in S$. Then $\operatorname{WSC}(\Gamma) \neq \emptyset$.

Proof. Let $\pi: N \rightarrow N$ be a permutation, assigning rank number $\pi(i)$ to any player $i \in N$. For a player $i \in N$, we define $\pi^{i}=\{j \in N \mid \pi(j) \leqslant \pi(i)\}$ as the set of predecessors of player $i$. For every $s \in S$, the marginal vector $m^{\pi}\left(\Gamma_{s}\right) \in \mathbb{R}^{N}$ is given by

$$
m^{\pi, i}\left(\Gamma_{s}\right)=v_{s}\left(\pi^{i}\right)-v_{s}\left(\pi^{i} \backslash\{i\}\right), \quad i \in N,
$$

and thus assigns to player $i$ his marginal contribution to the worth of the coalition consisting of all his predecessors in $\pi$. We show that $\bar{x}$ defined by $\bar{x}_{s}=m^{\pi}\left(\Gamma_{s}\right), s \in S$, belongs to WSC $(\Gamma)$.

Since $\Gamma_{s}$ is convex, it holds that $\bar{x}_{s} \in \mathrm{C}\left(\Gamma_{S}\right)$ for all $s \in S$ [12]. Using Theorem 4.4, it remains to be shown that there is no $C \subset N$ and allocation $x^{C}$ such that $x_{s}^{C} \in \mathrm{C}\left(\Gamma_{s}, C\right)$ for all $s \in S$, and $u^{i}\left(x^{i}\right)>u^{i}\left(\bar{x}^{i}\right)$ for all $i \in C$.

Consider $C \subset N$ and $x^{C}$ with $x_{s}^{C} \in \mathrm{C}\left(\Gamma_{s}, C\right)$ for all $s \in S$. Let $i$ be the player in $C$ with the highest value of $\pi(i)$. It holds that

$$
x_{s}^{i} \leqslant v_{s}(C)-v_{s}(C \backslash\{i\}) \leqslant v_{s}\left(\pi^{i}\right)-v_{s}\left(\pi^{i} \backslash\{i\}\right)=\bar{x}_{s}^{i},
$$

where the first inequality follows since $x_{s}^{C} \in \mathrm{C}\left(\Gamma_{s}, C\right)$ and the second inequality since by the choice of $i$ as the highest ranked player in $C$ according to $\pi$ it holds that $C \backslash\{i\} \subset \pi^{i} \backslash\{i\}$ and $\Gamma_{S}$ is convex. By monotonicity of $u^{i}$ we have that $u^{i}\left(x^{i}\right) \leqslant u^{i}\left(\bar{x}^{i}\right)$, which completes the proof.

In the proof of Theorem 5.3 we construct an allocation in the Weak Sequential Core by fixing a permutation and allocating the payoffs in each state by means of the corresponding marginal vector. Convexity of $\Gamma_{s}$ implies that all marginal vectors belong to the Core of $\Gamma_{s}$. Convexity is used once more to demonstrate that the highest ranked player in a deviating coalition gets less payoff in each state than in the original allocation. 
An interesting feature of Theorem 5.3 is that we do not need to make additional assumptions on the utility functions of the players. Within the framework of expected utility, we allow for both risk-averse and risk-loving players. Also many theories of non-expected utility maximization are covered by our result. This is in contrast to the classical definition of the Core, which might be empty-valued under the same assumptions. Considering the lack of results on non-emptiness of the Weak Sequential Core in the literature so far, this comes as a surprise.

The idea of fixing a certain permutation in the proof, might suggest that the convexity assumption could be weakened to permutational convexity; i.e. assuming that each TU-game $\Gamma_{S}$ is permutationally convex with respect to the same permutation $\pi$. Note however, that this weaker assumption holds for the TUU-game presented in Example 5.1 for the permutation $\pi=(3,2,1)$, whereas the Weak Sequential Core of that game is empty.

We show next that it is not possible to weaken convexity to exactness, a property introduced in [11].

Definition 5.4. A TU-game $(N, v)$ is exact if for all $C \subset N$ there exists a core allocation $x$ such that $x(C)=v(C)$.

For TU-games with three players, the notion of convexity coincides with the one of exactness. For games with more than three players, exactness is a weaker property. To verify whether a game is exact, we use the notion of exact balancedness, introduced in [3]. For $C \subset N, a(C) \in \mathbb{R}^{N}$ is the membership vector in $C$, where $a_{i}(C)=1$ if $i \in C$ and $a_{i}(C)=0$ otherwise.

Definition 5.5. An exactly balanced vector of weights is a vector $\left(\lambda_{C}\right)_{C \in \mathcal{N}}$ such that $\lambda_{D} \in \mathbb{R}$ for some $D \in \mathcal{N}$, and $\lambda_{C} \in \mathbb{R}_{+}$for all $C \neq D$, and $\sum_{C \in \mathcal{N}} \lambda_{C} a(C)=a(N)$. A TU-game $(N, v)$ is exactly balanced if $\sum_{C \in \mathcal{N}} \lambda_{C} v(C) \leqslant v(N)$ for all exactly balanced vectors of weights.

The following result was shown in [3].

Theorem 5.6. A TU-game $(N, v)$ is exact if and only if it is exactly balanced.

The only difference to the condition of balancedness is that one weight, $\lambda_{D}$, is not restricted to be non-negative. Since this creates an extra degree of freedom, the set of exactly balanced vectors is larger than the set of balanced vectors, so the class of exactly balanced games is a subset of the class of balanced games. Exact balancedness can also be used to give any easy proof of the fact that the class of exactly balanced games is a subset of the class of totally balanced games.

The following example shows that the Weak Sequential Core can be empty when all the games $\Gamma_{s}$ are exact.

Example 5.7. Consider a TUU-game $\Gamma$ with five players and six future states, all occurring with equal probability. All players are strictly risk-averse von Neumann-Morgenstern utility maximizers. We consider the vectors $w^{1}=(2,1,3,0,2)$ and $w^{2}=(0,5,1,2,0)$, and we define a TU-game $(N, w)$ with $N=\{1, \ldots, 5\}$ by

$$
w(C)=\min \left\{w^{1}(C), w^{2}(C)\right\}, \quad C \subset N .
$$

We define the permutations $\pi_{1}, \ldots, \pi_{6}$ by

$$
\begin{array}{lll}
\pi_{1}=(1,2,3,4,5), & \pi_{3}=(3,1,2,4,5), & \pi_{5}=(2,3,1,4,5), \\
\pi_{2}=(1,2,3,5,4), & \pi_{4}=(3,1,2,5,4), & \pi_{6}=(2,3,1,5,4),
\end{array}
$$


and, for $s=1, \ldots, 6$, we define the TU-game $\Gamma_{s}=\left(N, v_{s}\right)$ by setting

$$
v_{s}(C)=w\left(\pi_{s}(C)\right), \quad C \subset N,
$$

resulting in a TUU-game $\Gamma$ with five players and six states.

The TU-games $\left(N, v_{s}\right)$ are all obtained by renaming the players in the TU-game $(N, w)$. All properties derived for $(N, w)$ thereby immediately carry over to the games $\left(N, v_{s}\right)$ by taking appropriate permutations. We first analyze $(N, w)$. We show that $(N, w)$ is exact. By Definition 5.5 we have to check exact balancedness. Consider a vector of balancing weights $\left(\lambda_{C}\right)_{C \in \mathcal{N}}$. If all the balancing weights are non-negative, then

$$
\sum_{C \in \mathcal{N}} \lambda_{C} w(C) \leqslant \min \left\{\sum_{C \in \mathcal{N}} \lambda_{C} w^{1}(C), \sum_{C \in \mathcal{N}} \lambda_{C} w^{2}(C)\right\} \leqslant \min \left\{w^{1}(N), w^{2}(N)\right\}=w(N),
$$

where the second inequality follows from the additivity of the TU-games derived from $w^{1}$ and $w^{2}$. If one of the balancing weights is negative, say $\lambda_{D}$, then assume without loss of generality that $w(D)=w^{1}(D)$. We have that

$$
\begin{aligned}
\sum_{C \in \mathcal{N} \backslash\{D\}} \lambda_{C} w(C) & \leqslant \min \left\{\sum_{C \in \mathcal{N} \backslash\{D\}} \lambda_{C} w^{1}(C), \sum_{C \in \mathcal{N} \backslash\{D\}} \lambda_{C} w^{2}(C)\right\} \\
& \leqslant \min \left\{w^{1}(N)+\lambda_{D} w^{1}(D), w^{2}(N)+\lambda_{D} w^{2}(D)\right\}=w(N)+\lambda_{D} w(D) .
\end{aligned}
$$

We next compute the Core $\mathrm{C}(N, w)$. It is immediate to verify that $w^{1}, w^{2} \in \mathrm{C}(N, w)$, as well as all convex combinations $\mu w^{1}+(1-\mu) w^{2}$, where $\lambda \in[0,1]$. Choices for $\mu$ below 0 or above 1 lead to allocations outside the Core. We show next that $\mathrm{C}(N, w)$ is contained in a 1-dimensional space, leading to the characterization of the Core of $(N, w)$ as the convex hull of $w^{1}$ and $w^{2}$.

Let $x \in \mathrm{C}(N, w)$. Since $x(N)=w(N)$, we have that

$$
x_{1}+x_{2}+x_{3}+x_{4}+x_{5}=8 .
$$

Since $w(\{1,2,3\})=6$ and $w(\{4,5\})=2$, we find that

$$
x_{4}+x_{5}=2 .
$$

It holds that

$$
\begin{aligned}
& x_{3}+x_{4}=3, \\
& x_{2}+x_{3}+x_{5}=6,
\end{aligned}
$$

since $w(\{1,2,5\})=5$ and $w(\{3,4\})=3$, and $w(\{1,4\})=2$ and $w(\{2,3,5\})=6$, respectively. Since (2)-(5) are four independent equations in five unknowns, we have shown that the Core of $(N, w)$ is contained in a 1-dimensional space.

Consider $\bar{x} \in \operatorname{WSC}(\Gamma)$. By Theorem 4.4 it holds that $\bar{x}_{s} \in \Gamma_{s}$ for all $s \in S$. Moreover, there is no $C \subset N$ and allocation $x^{C}$ such that $x_{s}^{C} \in \mathrm{C}\left(\Gamma_{s}, C\right)$ for all $s \in S$, and $u^{i}\left(x^{i}\right)>u^{i}\left(\bar{x}^{i}\right)$ for all $i \in C$. Suppose there are states $s, s^{\prime} \in S$ such that $\bar{x}_{s}^{4} \neq \bar{x}_{s^{\prime}}^{4}$. Take $C=\{4,5\}$. Since $\bar{x}_{s}^{4}+\bar{x}_{s}^{5}=2$ for all $s \in S$, it holds that

$$
x_{\bar{s}}^{C}:=\left(\frac{1}{6} \sum_{s \in S} \bar{x}_{s}^{4}, \frac{1}{6} \sum_{s \in S} \bar{x}_{s}^{5}\right) \in \mathrm{C}\left(\Gamma_{\bar{s}}, C\right), \quad \bar{s} \in S .
$$


Because players 4 and 5 are strictly risk-averse, we have that $u^{4}\left(x^{4}\right)>u^{4}\left(\bar{x}^{4}\right)$ and $u^{5}\left(x^{5}\right)>$ $u^{5}\left(\bar{x}^{5}\right)$, a contradiction to $\bar{x} \in \mathrm{WSC}(\Gamma)$. Consequently, it holds that $\bar{x}_{s}^{4}$ and $\bar{x}_{s}^{5}$ are independent of $s$.

We define $\alpha \in[0,2]$ to be equal to $\bar{x}_{s}^{4}$. Since $\mathrm{C}\left(\Gamma_{s}\right)$ is the convex hull of $w^{1}$ and $w^{2}$ up to the permutation $\pi_{s}$, we find that $\bar{x}^{1}=(2-\alpha, \alpha, 3-\alpha, 1+\alpha, 1+2 \alpha, 5-2 \alpha)$. Moreover, $\bar{x}^{2}$ and $\bar{x}^{3}$ are identical to $\bar{x}^{1}$, up to a permutation. For $C=\{1,2,3\}$, we define the allocation $x^{C}$ by $x_{s}^{C}=(2,2,2)$ for all $s \in S$. It is easily verified that $(2,2,2) \in \mathrm{C}\left(\Gamma_{s}, C\right)$ for all $s \in S$. Since the players in $C$ are strictly risk averse, we have that $u^{i}\left(x^{i}\right)>u^{i}\left(\bar{x}^{i}\right)$ for all $i \in C$. By Theorem 4.4 this contradicts $\bar{x} \in \mathrm{WSC}(\Gamma)$.

We have shown that $\operatorname{WSC}(\Gamma)=\emptyset$.

\section{Conclusion}

In this paper we have introduced uncertainty into transferable utility games. Since in reality most surplus sharing decisions are made under uncertainty, this is a natural and important extension. It is not straightforward though, how to define an appropriate core concept for this stochastic setting. In this paper we consider allocations that are stable in the absence of commitment possibilities. These requirements lead to the notion of credibility. A credible deviation is self-enforcing in the sense that a coalition can credibly deviate from a given allocation if no sub-coalition ever has a credible counter-deviation. These considerations lead to the definition of the Weak Sequential Core.

We have an easy characterization of the Weak Sequential Core. All allocations in the Weak Sequential Core belong to the Core of the transferable utility game played after the resolution of uncertainty. Moreover, no coalition can block an allocation in the Weak Sequential Core ex ante by means of an allocation that belongs to the Core of all the ex post games reduced to the coalition. This property facilitates the application of the concept and the proof of its nonemptiness. We show that convexity of the ex post games is sufficient for the non-emptiness of the Weak Sequential Core, but that convexity cannot be weakened to permutational convexity or exactness.

\section{References}

[1] B.D. Bernheim, B. Peleg, M. Whinston, Coalition-proof Nash equilibria I. Concepts, J. Econ. Theory 42 (1987) $1-12$.

[2] W. Bossert, J. Derks, H. Peters, Efficiency in uncertain cooperative games, Math. Soc. Sci. 50 (1) (July 2005) 12-23.

[3] P. Csóka, P.J.-J. Herings, L.A. Kóczy, Balancedness conditions for exact games, Math. Methods Oper. Res. (2011), doi:10.1007/s00186-011-0348-3, forthcoming.

[4] D.B. Gillies, Solutions to general non-zero-sum games, Ann. of Math. Stud. 40 (1959) 47-85.

[5] D. Granot, Cooperative games in stochastic characteristic function form, Manage. Sci. 23 (6) (February 1977) 621630.

[6] H. Habis, P.J.-J. Herings, A note on the weak sequential core of dynamic TU games, Int. Game Theory Rev. 12 (4) (December 2010) 407-416.

[7] L. Kranich, A. Perea, H. Peters, Core concepts for dynamic TU games, Int. Game Theory Rev. 7 (1) (2005) $43-61$.

[8] A. Predtetchinski, The strong sequential core for stationary cooperative games, Games Econ. Behav. 61 (1) (October 2007) 50-66.

[9] A. Predtetchinski, P.J.-J. Herings, A. Perea, The weak sequential core for two-period economies, Int. J. Game Theory 34 (1) (April 2006) 55-65.

[10] D. Ray, Credible coalitions and the core, Int. J. Game Theory 18 (2) (1989) 185-187.

[11] D. Schmeidler, Cores of exact games, J. Math. Anal. Appl. 40 (1972) 214-225.

[12] L.S. Shapley, Cores of convex games, Int. J. Game Theory 1 (1) (1971) 11-26. 
[13] J. Suijs, P. Borm, Stochastic cooperative games: Superadditivity, convexity, and certainty equivalents, Games Econ. Behav. 27 (2) (May 1999) 331-345.

[14] J. Suijs, P. Borm, A. De Waegenaere, S. Tijs, Cooperative games with stochastic payoffs, Europ. J. Operational Res. 113 (1) (February 1999) 193-205.

[15] R. Vohra, Incomplete information, incentive compatibility, and the core, J. Econ. Theory 86 (1999) $123-147$. 